\title{
Uniqueness of the Translationally Invariant Ground State in Quantum Spin Systems
}

T. Matsui ${ }^{\star}$

Department of Mathematics and Computer Science, University College of Swansea, Singleton Park, Swansea SA2 8PP, Wales, UK

Abstract. We introduce a class of quantum spin systems on $\mathbb{Z}^{d}$. We show that the translationally invariant ground state is unique for this system if it is in a strong external field.

1.

In this paper, we study translationally invariant ground states of quantum spin systems. We consider our problems in $C^{*}$ algebraic framework. (See [2].) We introduce a class of quantum spin Hamiltonians which are translationally invariant and have the Perron Frobenius property. The Hamiltonians contain an external field term. We establish uniqueness of translationally invariant ground state if the strength of the external field is sufficiently large. Our class of Hamiltonian contains Quantum Ising models and Heisenberg models. In the due course of the proof, we will see that the invariant ground states of our systems can be realized by (finite temperature) Gibbs states of classical spin models. This correspondence has been first found by Kirkwood and Thomas in [4].

To be more precise, we introduce some notations. Let $\sigma_{\alpha}(\alpha=x, y, z)$ be Pauli spin matrices,

$$
\sigma_{x}=\left[\begin{array}{ll}
0 & 1 \\
1 & 0
\end{array}\right], \quad \sigma_{y}=\left[\begin{array}{cc}
0 & -i \\
i & 0
\end{array}\right], \quad \sigma_{z}=\left[\begin{array}{rr}
1 & 0 \\
0 & -1
\end{array}\right]
$$

We consider the $C^{*}$ algebra $\mathscr{A}=\bigotimes_{\mathbb{Z}^{d}} M_{2}(\mathbb{C})$ and by $\sigma_{\alpha}^{(j)}\left(j \in \mathbb{Z}^{d}, \alpha=x, y, z\right)$ we denote the Pauli spin matrix on site $j$. Hence $\sigma_{\alpha}^{(j)}$ satisfies the following commutation relations:

$$
\begin{aligned}
{\left[\sigma_{\alpha}^{(j)}, \sigma_{\beta}^{(k)}\right] } & =\sigma_{\alpha}^{(j)} \sigma_{\beta}^{(k)}-\sigma_{\beta}^{(k)} \sigma_{\alpha}^{(j)}=0, \text { if } k \neq j, \\
\sigma_{\alpha}^{(j)} \sigma_{\beta}^{(j)} & =i \varepsilon_{\alpha \beta \gamma} \sigma_{\gamma}^{(j)},
\end{aligned}
$$

where $\varepsilon_{\alpha \beta \gamma}$ is the totally antisymmetric tensor with $\varepsilon_{x y z}=1$.

\footnotetext{
* Supported by the Science and Engineering Research Council
} 
Let $\mathscr{A}_{\text {loc }}$ be the subalgebra of strictly local elements, that is, the set of all polynomials of $\sigma_{\alpha}^{(j)}$.

We next define the lattice translation automorphism $\alpha_{k}\left(k \in \mathbb{Z}^{d}\right)$ via the formula,

$$
\alpha_{k}\left(\sigma_{\alpha}^{(j)}\right)=\sigma_{\alpha}^{(j+k)} .
$$

The Hamiltonian we consider has the following form:

$$
H(\lambda)=-\left\{\lambda \sum_{j \in \mathbb{L}^{d}} \sigma_{x}^{(j)}+\sum_{j \in \mathbb{L}^{d}} V_{j}\left(\sigma_{x}, \sigma_{z}\right)\right\},
$$

where $\lambda$ is a real parameter and $V_{j}\left(\sigma_{x}, \sigma_{z}\right)$ is a selfadjoint element of $\mathscr{A}_{\text {loc }}$.

The time evolution is determined via the formula,

$$
\gamma_{t}(Q)=e^{i t H} Q e^{-i t H} \text { for } Q \text { in } \mathscr{A} .
$$

Definition 1.1. A state $\omega$ of $\mathscr{A}$ is an invariant ground state of $H(\lambda)$ if $\omega$ satisfies the following two conditions:

(i) $\omega \circ \alpha_{k}(Q)=\omega(Q) \quad$ for $k$ in $\mathbb{Z}^{d}$, and $Q$ in

(ii) $\omega\left(Q^{*}[H(\lambda), Q]\right) \geqq 0$ for $Q$ in $\mathscr{A}_{\text {loc }}$.

We make the following assumptions for $V_{j}\left(\sigma_{x}, \sigma_{z}\right)$ in (1.4).

Assumption 1.2.

(i) (translational invariance)

$$
\alpha_{k}\left(V_{j}\left(\sigma_{x}, \sigma_{z}\right)\right)=V_{j+k}\left(\sigma_{x}, \sigma_{z}\right) \text { for any } j \text { and } k \text { in } \mathbb{Z}^{d} .
$$

(ii) (Perron Frobenius Property). Let $A, B$ be finite subsets of $\mathbb{Z}^{d}$ and we set

$$
\sigma_{x}(A)=\prod_{j \in A} \sigma_{x}^{(j)}, \quad \sigma_{z}(B)=\prod_{j \in B} \sigma_{z}^{(j)} .
$$

Then

$$
V_{j}\left(\sigma_{x}, \sigma_{z}\right)=\sum_{A, B \subset \mathbb{Z}^{d}} V_{A, B}^{(j)} \sigma_{x}(A) \sigma_{z}(B),
$$

where

(a) $\sum_{B \subset \mathbb{Z}^{d}} V_{A, B}^{(j)} \sigma_{z}(B)=V_{A}^{(j)}\left(\sigma_{z}\right) \geqq 0$

if $A$ is not empty. (The positivity of (1.9b) is the operator positivity.)

(b) $V_{A, B}^{(j)}=0$

\section{if $\# A \cap B$ is odd}

$\# A \cap B$ is the number of lattice sites in $A \cap B$.

Remark. In the representation (1.1) of Pauli matrices, (1.9b) and (1.9c) lead the reality and non-negativity of off-diagonal matrix elements in $V_{j}\left(\sigma_{x}, \sigma_{z}\right)$. We will see that the Hamiltonian $-H_{\Lambda}(\lambda)$ defined on a finite region $\Lambda$ in $\mathbb{Z}^{d}$ satisfies the positivity and irreducibility conditions of the Perron Frobenius theorem. Hence the system on a finite region $\Lambda$ has the Unique ground state. However, in the 
infinite volume limit, we can't expect uniqueness of the ground state. If fact for the exactly solvable 1 dimensional quantum Ising model, it has been rigorously established that in the strong field the ground state is non-unique.

Theorem 1.3. Suppose Assumption 1.2 for $V_{j}\left(\sigma_{x}, \sigma_{z}\right)$ is satisfied. Then there exists $\lambda_{0}>0$ such that for $\lambda>\lambda_{0}$ the translationally invariant ground state of $H(\lambda)$ is unique.

Examples

(i) Quantum Ising model. The Hamiltonian of this model is

$$
H(\lambda)=-\lambda \sum_{j \in \mathbb{Z}^{a}} \sigma_{x}^{(j)}+\sum_{j \in \mathbb{Z}^{a}} V_{j}\left(\sigma_{z}\right),
$$

where $V_{j}\left(\sigma_{z}\right)$ is a polynomial of $\sigma_{z}^{(k)}\left(k \in \mathbb{Z}^{d}\right)$.

Let $\gamma_{t}^{(2)}$ be the time evolution automorphism defined by (1.5) and (1.9). Then

$$
\gamma_{t}^{(\lambda)}=\gamma_{t}^{(-\lambda)} \circ \operatorname{Ad}\left(\sum_{j \in \mathbb{Z}^{d}} \sigma_{z}^{(j)}\right) .
$$

By Theorem 1.3 and (1.11), we have uniqueness of the invariant ground state for both positive and negative large $\lambda$.

(ii) Heisenberg model. The Hamiltonian is determined by

$$
H(\lambda)=-\left\{\lambda \sum_{j \in \mathbb{L}^{d}} \sigma_{x}^{(j)}+\sum_{\left|j-J^{\prime}\right|=1}\left(\alpha_{x} \sigma_{x}^{(j)} \sigma_{x}^{\left(j^{\prime}\right)}+\alpha_{y} \sigma_{y}^{(j)} \sigma_{y}^{\left(j^{\prime}\right)}+\alpha_{z} \sigma_{z}^{(j)} \sigma_{z}^{\left(j^{\prime}\right)}\right)\right\} .
$$

Our method covers the following cases.

$$
\text { (a) } \alpha_{x}<0, \alpha_{y}>0, \alpha_{z}<0, \text { (b) } \alpha_{x}<0, \alpha_{y}<0, \alpha_{z}>0 \text {. }
$$

To see this it suffices to note the following identity:

$$
a\left(1+b \sigma_{x}^{(j)} \sigma_{x}^{\left(j^{\prime}\right)}\right)\left(1+c \sigma_{z}^{(j)} \sigma_{z}^{\left(j^{\prime}\right)}\right)=a+a b \sigma_{x}^{(j)} \sigma_{x}^{\left(j^{\prime}\right)}-a b c \sigma_{y}^{(j)} \sigma_{y}^{\left(j^{\prime}\right)}+a c \sigma_{z}^{(j)} \sigma_{z}^{\left(j^{\prime}\right)} .
$$

We now sketch briefly the idea of the proof of our main theorem. Our proof depends crucially on ideas of Kirkwood and Thomas in [4]. We consider a perturbation times a parameter $\delta$ to the Hamiltonian. If the ground state energy density is differentiable at $\delta=0$ for any perturbation, then the translationally invariant ground state is unique. (See Proposition 2.1.) By the result of [4], this energy density is differentiable for a restricted class of local observables. To prove uniqueness, we consider a realization of the translationally invariant ground state which is again due to Kirkwood and Thomas in [4]. In [4] exponential decay of correlations is established for observable generated by diagonal matrices. What we will do is to study the representation of the set of all observables using $C^{*}$ algebraic techniques.

2.

Let $\Lambda$ be a subset of $\mathbb{Z}^{d}$, and $\mathscr{A}_{\Lambda}$ be the $C^{*}$ subalgebra generated by $\sigma_{\alpha}^{(j)}(\alpha=x, y, z, j$ is in $\Lambda$ ). We consider the Hamiltonian $H$ and the local Hamiltonian $H_{\Lambda}$ defined by 


$$
\begin{aligned}
H & =\sum_{X \subset \mathbb{Z}^{d}} \Phi(X), \\
H_{\Lambda} & =\sum_{X \cap \Lambda \neq \Phi} \Phi(X),
\end{aligned}
$$

where the sum in (2.1) is taken over all finite subsets of $\mathbb{Z}^{d}$ and $\Phi(X)$ is a selfadjoint element of $\mathscr{A}_{\Lambda}$.

Let $\omega$ be an invariant state of $\mathscr{A}$, i.e. $\omega$ is a state satisfying $\omega \circ \alpha_{k}=\omega$ for all $k$ in $\mathbb{Z}^{d}$ If we assume translation invariance of $H$ and

$$
\sum_{X \ni 0}\|\Phi(X)\|<\infty
$$

then the following limit exists:

$$
H(\omega)=\lim _{\Lambda \rightarrow \infty} \frac{\omega\left(H_{\Lambda}\right)}{|\Lambda|} .
$$

(The limit must be taken in the sense of van Hove, see [2].) Furthermore the following identity is valid.

$$
H(\omega)=\sum_{X \ni 0} \frac{\omega(\Phi(X))}{|X|} .
$$

We call $H(\omega)$ the mean energy of the state $\omega$.

The result below is proved by O. Bratteli, A. Kishimoto and D. Robinson in [1].

Theorem. Let $\omega$ be an invariant state. The following conditions are equivalent.

(i) $\omega$ is a ground state for $H$.

(ii) $\omega$ minimizes $H(\omega)$.

We now define the mean ground state energy $E(H)$ by

$$
E(H)=\inf _{\omega: \text { invariant state }} H(\omega) .
$$

Let $Q$ be a selfadjoint element of $\mathscr{A}$ and consider the perturbed Hamiltonian $H(\delta)$ determined via the equation,

$$
H(\delta)=H+\delta \sum_{k \in \mathbb{Z}^{d}} \alpha_{k}(Q) .
$$

Proposition 2.1. If $E(H(\delta))$ is differentiable at $\delta=0$, for any invariant ground state $\omega$, the following identity is valid:

$$
\left.\frac{d}{d \delta} E(H(\delta))\right|_{\delta=0}=\omega(Q)
$$

Proof. This is a consequence of the theorem cited above. Let $\omega$ be an invariant ground state of $H$. Then by (2.4),

$$
E(H(\delta)) \leqq \omega\left(\sum_{X \in 0} \frac{\Phi(X)}{|X|}+\delta Q\right)=\sum_{X \in O} \frac{\omega(\Phi(X))}{|X|}+\delta \omega(Q)=E(H)+\delta \omega(Q) .
$$


If we set $\delta$ positive and take $\delta$ to zero,

$$
\left.\frac{d}{d \delta} E(H(\delta))\right|_{\delta=0} \leqq \omega(Q) .
$$

If we set $\delta$ negative

$$
\left.\frac{d}{d \delta} E(H(\delta))\right|_{\delta=0} \geqq \omega(Q) .
$$

By the result of Sect. 3, we will establish the differentiability for $Q=\sigma_{z}(B)$, $\sigma_{z}(A) \sigma_{x}^{(j)}$ and $\sigma_{x}^{(j)}$. (q.e.d.)

The Hamiltonian we introduced in Sect. 1 is real in the following sense. Let $j(\cdot)$ be the complex conjugation of $\mathscr{A}$ defined by

$$
j\left(\sigma_{x}^{(k)}\right)=\sigma_{x}^{(k)}, \quad j\left(\sigma_{y}^{(k)}\right)=-\sigma_{y}^{(k)}, \quad j\left(\sigma_{z}^{(k)}\right)=\sigma_{z}^{(k)} .
$$

and

$$
j(c Q)=\bar{c} j(Q) \text { if } \quad c \in \mathbb{C}, \quad Q \in j\left(Q_{1} Q_{2}\right)=j\left(Q_{1}\right) j\left(Q_{2}\right) .
$$

Then $j \circ \gamma_{t}(Q)=\gamma_{t} \circ j(Q)$ for $Q$ in $\mathscr{A}$, where $\gamma_{t}$ is defined in (1.5).

Definition 2.2. A state $\omega$ is $j$ symmetric if for any $Q$ in $\mathscr{A}$

$$
\omega(j(Q))=\overline{\omega(Q)}
$$

\section{Lemma 2.3.}

(i) Let $\omega_{0}$ be a ground state of the 'real' Hamiltonian, i.e. $j\left(H_{\Lambda}\right)=H_{\Lambda}$ for any finite 1. Set

$$
\omega(Q)=\frac{1}{2}\left\{\omega_{0}(Q)+\omega_{0}(j(Q))\right\} .
$$

Then $\omega$ is a jymmetric ground state.

(ii) A state $\omega$ is $j$ symmetric if and only if

$$
\omega\left(\sigma_{x}(A) \sigma_{z}(B)\right)=0
$$

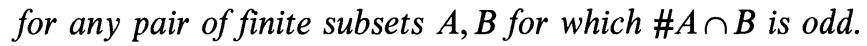

3.

In this section we give estimates of the ground state energy. We apply the expansion in $\lambda$ developed in [4]. This method is quite simple and useful for our purpose.

We start with the Hamiltonian on a finite cube $\Lambda$ with volume $L^{d}$, and we impose a periodic boundary condition, so we set

$$
\sigma_{\alpha}^{\left(j+l_{k}\right)}=\sigma_{\alpha}^{(j)}
$$

for $j$ in $\Lambda$ and $\alpha=x, y, z, l_{k}=(0,0, \ldots, 1, \ldots, 0) \in \mathbb{Z}^{d}$. 
Then consider the perturbed Hamiltonian defined by

$$
\tilde{H}_{\Lambda}(\varepsilon, \delta)=-\left\{\sum_{j \in \Lambda}\left(\sigma_{x}^{(j)}+\varepsilon V_{j}\left(\sigma_{x}, \sigma_{z}\right)+\delta \alpha_{j}(Q)\right\},\right.
$$

where $V_{j}\left(\sigma_{x}, \sigma_{z}\right)$ satisfies Assumption 1.2,

$$
V_{j}\left(\sigma_{x}, \sigma_{z}\right)=\sum_{A, B} V_{A, B}^{(j)} \sigma_{x}(A) \sigma_{z}(B)
$$

We consider the following case:

$$
Q=\left\{\begin{array}{l}
\sigma_{x}^{(0)} \\
\left\{1 \pm \sigma_{z}(A)\right\} \sigma_{x}^{(0)} \\
\sigma_{z}(B)
\end{array}\right.
$$

where $A$ and $B$ are finite subsets and $A$ does not contain the origin 0 .

We fix a representation of Pauli matrices as in (1.1) and use the following basis $|\sigma\rangle$

$$
|\sigma\rangle=\bigotimes_{j \in \Lambda} e_{j}, \quad e_{j}=\left(\begin{array}{l}
1 \\
0
\end{array}\right) \text { or } \quad\left(\begin{array}{l}
0 \\
1
\end{array}\right)
$$

We identify $|\sigma\rangle$ with a configuration of classical spin in $\{1,-1\}^{\Lambda}$.

In this basis, we may apply the Perron Frobenius theorem for $-\tilde{H}_{\Lambda}(\varepsilon, \delta)$. (See [3] for the criterion of this theorem.)

Lemma 3.1. The Hamiltonian $-\tilde{H}_{\Lambda}(\varepsilon, \delta)$ satisfies the assumptions for the Perron Frobenius theorem (irreducibility and non-negativity) if $\varepsilon$ is non-negative and $\delta$ is also non-negative in the case (3.3a) (3.3b) or if $\varepsilon$ is non-negative in the case (3.3c).

Proof. It suffices to show

$$
\left\langle\sigma^{\prime}\left|e^{-t \tilde{H}_{\Lambda}(\varepsilon, \delta)}\right| \sigma\right\rangle>0
$$

for $t$ positive and any vectors $|\sigma\rangle\left|\sigma^{\prime}\right\rangle$ of the form (3.4). It is easy to show the inequality by

$$
\left\langle\sigma^{\prime}\left|e^{-t \hat{H}_{\Lambda}^{(\varepsilon, \delta)}}\right| \sigma\right\rangle \geqq C\left\langle\sigma^{\prime}\left|e^{t \sum_{j \in \Lambda} \sigma_{x}^{(j)}}\right| \sigma\right| \geqq C(\sinh t)^{|\Lambda|},
$$

because the assumption (1.9b) ensures the non-negativity of matrix elements of $V_{j}\left(\sigma_{x}, \sigma_{z}\right)$ after adding a large positive scalar to $-\widetilde{H}_{\Lambda}(\varepsilon, \delta)$. (q.e.d.)

By this result $H_{\Lambda}(\varepsilon, \delta)$ has a unique ground state and all the coefficients of this ground state are positive. We also note that this state and the positive vector are periodic by uniqueness of the Perron Frobenius vector.

The positivity of the ground state vector enables us to use the following definition:

$$
\begin{aligned}
-\tilde{H}_{\Lambda}(\varepsilon, \delta) \psi_{\Lambda}(\varepsilon, \delta) & =|\Lambda| e_{\Lambda}(\varepsilon, \delta) \psi_{\Lambda}(\varepsilon, \delta), \\
\psi_{\Lambda}(\varepsilon, \delta) & =\sum_{\sigma} e^{-1 / 2 h_{\Lambda}(\varepsilon, \delta)(\sigma)}|\sigma\rangle .
\end{aligned}
$$


Let $\mathscr{B}_{A}$ be the set of all functions of $\{1,-1\}^{\Lambda}$. Any function $f(\sigma)$ is a linear combination of monomials $\sigma(A)$, where $\sigma_{j}$ is the spin at the site $j$ taking value 1 or -1 and

$$
\sigma(A)=\prod_{j \in A} \sigma_{j}
$$

We can identify $\sigma_{j}$ and $\sigma_{z}^{(j)}, \sigma(A)$ and $\sigma_{z}(A)$. Thus we may write

$$
f(\sigma)=\sum_{A \subset \Lambda} f_{A} \sigma(A) .
$$

By (3.9) $\mathscr{B}_{\Lambda}$ can be regarded as a subalgebra of $\mathscr{B}_{\Lambda}$, if $\Lambda \subset \Lambda^{\prime}$. Let $\mathscr{B}_{\infty}$ be the inductive limit of $\mathscr{B}_{\Lambda}$ by this inclusion,

$$
\mathscr{B}_{\infty}=\lim \mathscr{B}_{\Lambda} \text {. }
$$

We now define two norms \|\|$_{\infty},\|\|$ on $\mathscr{B}_{\infty}$,

$$
\begin{gathered}
\|f(\sigma)\|_{\infty}=\sup _{\sigma \in\{1,-1\}^{\}^{d}}}|f(\sigma)|, \\
\|f(\sigma)\|=\sum_{A \subset \mathbb{Z}^{d}}\left|f_{A}\right| .
\end{gathered}
$$

Obviously the following (in)equalities are valid:

$$
\begin{aligned}
\|f(\sigma)\|_{\infty} & \leqq\|f(\sigma)\|, \\
\|\sigma(A) f(\sigma)\| & =\|f(\sigma)\| .
\end{aligned}
$$

By (3.14) we have

$$
\begin{aligned}
\left\|f_{1}(\sigma) f_{2}(\sigma)\right\| & \leqq \sum_{A}\left|f_{1, A}\right|\left\|\sigma(A) f_{2}(\sigma)\right\| \\
& =\left(\sum_{A}\left|f_{1, A}\right|\right)\left\|f_{2}(\sigma)\right\|=\left\|f_{1}(\sigma)\right\|\left\|f_{2}(\sigma)\right\| .
\end{aligned}
$$

Let $\mathscr{B}$ be the completion of $\mathscr{B}_{\infty}$ by the norm \|\|$_{\infty} . \mathscr{B}$ may be identified with the $C^{*}$ subalgebra of $\mathscr{A}$ generated by $\sigma_{z}^{(j)}\left(j \in \mathbb{Z}^{d}\right)$. The. identification is

$$
\sigma(A)=\sigma_{z}(A)
$$

Let $f(\sigma)$ be in $\mathscr{B}_{\infty}$ and $A$ be a finite subset of $\mathbb{Z}^{d}$. We define $f^{(j)}(\sigma)$ and $f^{A}(\sigma)$ by the following equations:

$$
\begin{aligned}
f^{(j)}(\sigma) & =\sum_{A \in j} f_{A} \sigma(A), \\
f^{A}(\sigma) & =\sum_{\#(B \cap A) \text { is odd }} f_{B} \sigma(B),
\end{aligned}
$$

where $f_{A}$ is the coefficient in (3.9). 
We first consider the case $\delta=0$. We set

$$
\tilde{H}_{\Lambda}(\varepsilon)=\tilde{H}_{\Lambda}(\varepsilon, \delta), e_{\Lambda}(\varepsilon)=e_{\Lambda}(\varepsilon, 0), h_{\Lambda}(\varepsilon)(\sigma)=h_{\Lambda}(\varepsilon, 0)(\sigma) .
$$

\section{Proposition 3.2.}

(i) There exists $\varepsilon_{0}, e_{\Lambda}(\varepsilon), h_{\Lambda}^{(j)}(\varepsilon)(\sigma), g(\varepsilon)$ such that $g(\varepsilon)$ is analytic in $\left\{|\varepsilon|<\varepsilon_{0}\right\}, g(0)=0$, $h_{\Lambda}(\varepsilon)(\sigma)$ is real and $e_{\Lambda}(\varepsilon), h_{\Lambda}(\varepsilon)(\sigma)$ satisfy $(3.7 a)$ and for $\varepsilon$ in $\left\{|\varepsilon|<\varepsilon_{0}\right\}$,

$$
\left|e_{\Lambda}(\varepsilon)\right|+\left\|h_{\Lambda}^{(j)}(\varepsilon)(\sigma)\right\| \leqq g(\varepsilon) .
$$

(ii) The following limits exist in the norm \|\| , and $h^{(j)}(\varepsilon)(\sigma)$ is analytic in $\left\{|\varepsilon|<\varepsilon_{0}\right\}$ :

$$
\begin{aligned}
& \lim _{\Lambda \rightarrow \infty} h_{\Lambda}^{(j)}(\varepsilon)(\sigma)=h^{(j)}(\varepsilon)(\sigma), \\
& \lim _{\Lambda \rightarrow \infty} h_{\Lambda}^{A}(\varepsilon)(\sigma)=h^{A}(\varepsilon)(\sigma) .
\end{aligned}
$$

Furthermore

$$
\left\|h^{A}(\varepsilon)(\sigma)\right\| \leqq|A| g(\varepsilon) .
$$

Proof. The main idea of proof is the same as that of [4].

(i) By use of the vector (3.4), (3.7) is written in the following form:

$$
\sum_{j \in \Lambda}\left\{e^{h_{\Lambda}^{(j)}(\varepsilon)(\sigma)}+\varepsilon\left(\sum_{A, B} V_{A, B}^{(j)} e^{h_{\Lambda}^{A}(\varepsilon)(\sigma)} \sigma(B)\right)-e_{\Lambda}(\varepsilon)\right\}=0 .
$$

We solve (3.23) by a perturbation expansion,

$$
\begin{aligned}
h_{\Lambda}^{(j)}(\varepsilon)(\sigma) & =\sum \tilde{h}_{n}^{(j)}(\sigma) \varepsilon^{n}, \\
e_{\Lambda}(\varepsilon) & =\sum \tilde{e}_{n} \varepsilon^{n},
\end{aligned}
$$

where we omit the subscript $\Lambda$ for $\tilde{h}_{n}^{(j)}$ and $\tilde{e}_{n}$ for simplicity of notations.

If $\varepsilon=0$, we can solve (3.7) easily and we see $h_{\Lambda}(0)(\sigma)$ is a constant, thus $\tilde{h}_{0}^{(j)}(\sigma)=0 \tilde{e}_{0}=1$. The expansions $(2.23),(3.24)$ lead to

$$
\begin{aligned}
\sum_{j \in \Lambda} & \left\{\tilde{h}_{n}^{(j)}(\sigma)+P_{n}\left(\tilde{h}_{1}^{(j)}, \ldots, \tilde{h}_{n-1}^{(j)}\right)-\tilde{e}_{n}\right\} \\
& =-\sum_{j \in \Lambda}\left\{\sum_{A, B} V_{A, B}^{(j)}\left(\tilde{h}_{n-1}^{A}(\sigma)+P_{n-1}\left(\tilde{h}_{1}^{A}(\sigma), \ldots, \tilde{h}_{n-2}^{A}(\sigma)\right)\right) \sigma(B)\right\},
\end{aligned}
$$

where $P_{n}\left(x_{1}, x_{2}, \ldots, x_{n-1}\right)$ is defined by

$$
\exp \left(\sum_{n=1}^{\infty} \varepsilon^{n} x_{n}\right)=1+\sum_{n=1}^{\infty}\left(x_{n}+P_{n}\left(x_{1}, \ldots, x_{n-1}\right)\right) \varepsilon^{n}
$$

Obviously all the coefficients in $P_{n}$ are positive. Equation (3.26) determines $\tilde{h}_{n}(\sigma)$, $\tilde{e}_{n}$ in the following sense. Let $f(\sigma)$ be a function written as

$$
f(\sigma)=\sum_{A} f_{A} \sigma(A)
$$


Equation (3.26) has the form

$$
c+\left(\sum_{j} f^{(j)}(\sigma)\right)=F(\sigma)
$$

where $F(\sigma)$ is a known function. However

$$
\left(\sum_{j} f^{(j)}(\sigma)\right)=\sum_{A \neq \Phi}|A| f_{A}
$$

Thus (3.28) determines the $f(\sigma)$ except the constant term $f_{\Phi}$ and the constant term of $F(\sigma)$ is equal to $C$. As the constant term in $h_{\Lambda}(\varepsilon)(\sigma)$ contributes to the normalization of the vector, (3.26) determines completely the ground state. (See $(3.7 b)$.)

Now it is easy to see

$$
\left\|f^{A}(\sigma)\right\| \leqq \sum_{j \in A}\left\|f^{(j)}(\sigma)\right\| .
$$

As we impose the periodic boundary condition, the following are valid:

$$
\begin{gathered}
\left\|\sum_{j \in A} \tilde{h}_{n}^{(j)}(\sigma)\right\|=|\Lambda|\left\|\tilde{h}_{n}^{(j)}(\sigma)\right\|, \\
\left\|\tilde{h}_{n}^{(A)}(\sigma)\right\| \leqq|A|\left\|\tilde{h}_{n}^{(j)}(\sigma)\right\| .
\end{gathered}
$$

Thus by (3.31) and (3.32), (3.26) implies that if $n \geqq 2$,

$$
\begin{aligned}
\left\|\tilde{h}_{n}^{(j)}(\sigma)\right\|+\left|\tilde{e}_{n}\right| \leqq & P_{n}\left(\left\|\tilde{h}_{1}^{(j)}(\sigma)\right\|, \ldots,\left\|\tilde{h}_{n-1}^{(j)}(\sigma)\right\|\right) \\
& +V\left\{c\left\|\tilde{h}_{n-1}^{(j)}(\sigma)\right\|+P_{n-1}\left(c\left\|\tilde{h}_{1}^{(j)}(\sigma)\right\|, \ldots\right)\right\},
\end{aligned}
$$

where

$$
\begin{aligned}
c & =\sup \left\{|A| ; V_{A, B}^{(j)} \neq 0\right\} \\
V & =\sum_{A, B}\left|V_{A, B}^{(j)}\right| .
\end{aligned}
$$

For $n=1$ we have

$$
\left\|\tilde{h}_{1}^{(j)}(\sigma)\right\|+\left|e_{1}\right| \leqq V .
$$

We now set

Then by (3.33)

$$
a_{n}=c\left\{\left\|\tilde{h}_{n}^{(j)}(\sigma)\right\|+\left|\tilde{e}_{n}\right|\right\}
$$

$$
\begin{aligned}
& a_{1} \leqq c V \\
& a_{n} \leqq c P_{n}\left(a_{1}, \ldots, a_{n-1}\right)+(V c)\left\{a_{n-1}+P_{n-1}\left(a_{1}, \ldots, a_{n-2}\right)\right\} .
\end{aligned}
$$

Let $f(\varepsilon)$ be a function defined implicitly by

$$
(1+c) f(\varepsilon)-(c V) \varepsilon-c\left\{e^{f(\varepsilon)}-1\right\}-(V c) \varepsilon\left\{e^{f(\varepsilon)}-1\right\}=0 .
$$

By the implicit function theorem, $f(\varepsilon)$ satisfying $f(0)=0$ exists and is real analytic in a neighbourhood of $\varepsilon=0$. Set 


$$
b_{n}=\left.\frac{1}{n !} \frac{d^{n}}{d \varepsilon} f(\varepsilon)\right|_{\varepsilon=0}
$$

Then $b_{n}$ satisfies

$$
\begin{aligned}
(1+c) b_{n} & =c\left\{b_{n}+P_{n}\left(b_{1}, \ldots, b_{n-1}\right)\right\}+(c V)\left\{b_{n-1}+P_{n-1}\left(b_{1}, \ldots, b_{n-2}\right)\right\}, \\
(1+c) b_{1} & =c V+c b_{1} .
\end{aligned}
$$

We can show the following inequality by induction and (3.37), (3.40) and (3.41):

Thus

$$
a_{n} \leqq b_{n}
$$

$$
\sum_{n=1} a_{n} \varepsilon_{n} \leqq f(\varepsilon)
$$

By setting $g(\varepsilon)=(1 / c) f(\varepsilon)$, we have (3.19).

(ii) By definition (3.18) we have

$$
\left\|h_{\Lambda}^{A}(\varepsilon)(\sigma)-h_{\Lambda^{\prime}}^{A}(\varepsilon)(\sigma)\right\| \leqq \sum_{j \in A}\left\|h_{\Lambda}^{(j)}(\varepsilon)(\sigma)-h_{\Lambda^{\prime}}^{(j)}(\varepsilon)(\sigma)\right\| .
$$

Thus (3.20b) and (3.21) follows from (3.20a). Suppose $\Lambda$ is larger than the range of potential $V_{j}\left(\sigma_{x}, \sigma_{z}\right)$. Then the same $\tilde{h}_{n}^{(j)}$ is the solution of (3.26) for different $\Lambda, \Lambda^{\prime} \Lambda \subset \Lambda^{\prime}$, if $n$ is small. So the following estimates can be proved:

$$
\left\|h_{\Lambda}^{(j)}(\varepsilon)-h_{\Lambda^{\prime}}^{(j)}(\varepsilon)\right\| \leqq c_{1} \varepsilon^{c_{2} L},
$$

where $L$ is the shortest side of $\Lambda$. This completes the proof. (q.e.d.)

Next we consider the Hamiltonian (3.2). By the choice of $Q$ in $(3.3),-\tilde{H}(\varepsilon, \delta)$ satisfies the assumption for the Perron Frobenius Theorem in the following range of parameter $\delta$ :

$$
\text { If } \begin{aligned}
Q= & \begin{cases}\sigma_{z}(B), & \delta: \text { arbitrary } \\
\sigma_{x}^{(0)}, & |\delta|<1\end{cases} \\
& \left\{1 \pm \sigma_{z}(A)\right\} \sigma_{x}^{(0)}, \quad \delta: \text { positive. }
\end{aligned}
$$

Proposition 3.3. Suppose $h_{\Lambda}(\varepsilon, 0)$ satisfies the following condition with a constant $d^{\prime}$ independent of $\Lambda$ :

$$
\left\|e^{h_{A}^{(j)}(\varepsilon, 0)(\sigma)}-1\right\|+|\varepsilon|\left\|\sum_{A, B} V_{A B}^{(j)} \sigma(B) e^{h_{0}^{A}(\varepsilon, 0)}\right\|<d^{\prime}<1 .
$$

Then there exists constants $\delta_{0}, c_{A}$, such that $e_{\Lambda}(\varepsilon, \delta)$ is an analytic function of $\delta$ in $\left\{|\delta|<\delta_{0}\right\}$, the limit $\lim _{\Lambda \rightarrow \infty} e_{\Lambda}(\varepsilon, \delta)$ exists

$$
\lim _{\Lambda \rightarrow \infty} e_{\Lambda}(\varepsilon, \delta)=e(\varepsilon, \delta)
$$

and

$$
\left\|h_{\Lambda}^{A}(\varepsilon, \delta)(\sigma)\right\|<c_{A}
$$


Remark 3.4. (i) (3.46) is satisfied if $\varepsilon$ is sufficiently small because the following estimate is valid by (3.15):

$$
\left\|e^{-h_{\Lambda}^{(j)(\varepsilon)(\sigma)}}-1\right\| \leqq e^{\| h_{\Lambda}^{(j)(\varepsilon)(\sigma) \|}}-1<1 .
$$

(ii) $e(\varepsilon, \delta)$ is analytic because for any sequence of analytic functions on a fixed domain, we can choose a subsequence convergent to an analytic function.

The proof of Proposition 3.3 goes along the same lines of Sect. 2 of [4]. We don't repeat it here.

4

We now set

$$
H(\lambda, \delta)=H(\lambda)-\delta \sum_{k \in \mathbb{Z}^{d}} \alpha_{k}(Q),
$$

where $Q$ is one of (3.3).

Proposition 4.1. Let $\varepsilon=(1 / \lambda)$ and $h_{\Lambda}^{(j)}(\varepsilon)(\sigma)$ satisfy (3.46).

(i) If $Q$ is either $\sigma_{z}(A)$ or $\sigma_{x}^{(j)}$, then the mean ground state energy $E(H(\lambda, \delta))$ is differentiable in $\delta$ at $\delta=0$.

(ii) If $Q=\left\{1 \pm \sigma_{z}(B)\right\} \sigma_{x}^{(j)}$, where $B$ does not contain $j, E(H(\lambda, \delta))$ is right differentiable at $\delta=0$.

Proof. It is easy to see

$$
\begin{gathered}
H(\lambda, \delta)=\lambda \tilde{H}\left(\frac{1}{\lambda} \frac{\delta}{\lambda}\right), \\
E(H(\lambda, \delta))=\lambda E\left(\tilde{H}\left(\frac{1}{\lambda} \frac{\delta}{\lambda}\right)\right)=\lambda e\left(\frac{1}{\lambda} \frac{\delta}{\lambda}\right) .
\end{gathered}
$$

Thus (i) follows from Proposition 3.3 and Remark 3.4 (iii). If we consider the case

$$
Q=\left\{1 \pm \sigma_{z}(B)\right\} \sigma_{x}^{(j)},
$$

we have (4.2b) for $\delta>0$. In view of (2.9a), it is easy to prove the assertion of (ii).

Proposition 4.1 and 2.1 imply the following.

Corollary 4.2. If $\lambda$ satisfy the condition of Proposition 4.1, for the invariant ground states $\omega_{1}, \omega_{2}$ of $H(\lambda)$, we have

$$
\begin{aligned}
\omega_{1}(Q) & =\omega_{2}(Q), \\
Q & =\sigma_{z}(A) \text { or } \sigma_{x}^{(j)} .
\end{aligned}
$$

Let $\mathscr{B}$ be the $C^{*}$ algebra generated by $\left\{\sigma_{z}^{(j)} j \in \mathbb{Z}^{d}\right\}$. As is remarked in Sect. 3, we may identify $\mathscr{B}$ with the function space $c\left(\{1,-1\}^{\mathbb{Z}^{d}}\right)$.

Let $F_{\lambda}$ be the linear map from $\mathscr{A}_{\text {loc }}$ to $\mathscr{B}$ defined by

$$
F_{\lambda}\left(\sigma_{z}(A) \sigma_{x}(B)\right)=\sigma(A) e^{h^{B}(\lambda)(\sigma)} .
$$


Let $F_{\lambda}^{(\Lambda)}$ be a linear map from $\mathscr{A}_{\Lambda}$ to $\mathscr{B}$ defined by

$$
F_{\lambda}^{(\Lambda)}\left(\sigma_{z}(A) \sigma_{x}(B)\right)=\sigma(A) e^{h_{\Lambda}^{B}(\lambda)(\sigma)}
$$

It is easy to see

$$
\begin{aligned}
F_{\lambda}^{(\Lambda)}\left(Q_{1} Q_{2}\right) & =Q_{1} F_{\lambda}^{(\Lambda)}\left(Q_{2}\right), \\
F_{\lambda}\left(Q_{1} Q_{2}\right) & =Q_{1} F_{\lambda}\left(Q_{2}\right), \text { if } Q_{1} \text { is in } \mathscr{B} .
\end{aligned}
$$

The vector state $\omega_{\Lambda}^{(\lambda)}(\cdot)$ associated with $\psi_{\Lambda}(1 / \lambda, 0)$ is the unique ground state of $H_{\Lambda}(\lambda)$. Now

$$
\omega_{\Lambda}^{(\lambda)}(Q)=\omega_{\Lambda}^{(\lambda)}\left(F_{\lambda}^{(\Lambda)}(Q)\right)
$$

To see this we consider the case

$$
Q=\sigma_{z}(A) \sigma_{x}(B)
$$

Then

$$
\omega_{\Lambda}^{(\lambda)}(Q)=\frac{1}{Z_{\Lambda}}\left\{\sum_{\sigma \sigma^{\prime}}\left\langle\sigma\left|\sigma_{z}(B)\right| \sigma^{\prime}\right\rangle e^{-(1 / 2) h_{\Lambda}(1 / \lambda)(\sigma)-(1 / 2) h_{\Lambda}(1 / \lambda)\left(\sigma_{A}^{\prime}\right)}\right\},
$$

where $Z_{A}$ is the normalization constant and $\sigma_{A}$ is the flip of spin in $A$.

By the definition (3.18), we have

$$
(4.7)=\frac{1}{Z_{\Lambda}}\left\{\sum_{\sigma} \sigma(B) e^{h_{\Lambda}^{A}(1 / \lambda)(\sigma)} e^{-h_{\Lambda}(1 / \lambda)(\sigma)}\right\} .
$$

Equation (4.8) may be interpreted as the integration of $F_{\Lambda}(Q)$ by a Gibbs measure.

We next consider the infinite volume limit.

Proposition 4.3. Suppose the limit (3.20) exists. Then we have the existence of the following:

$$
\omega^{(\lambda)}(Q)=\lim _{\Lambda \rightarrow \infty} \omega_{\Lambda}^{(\lambda)}(Q) \text { for } Q \in \mathscr{A}_{1 \mathrm{loc}}
$$

Furthermore

$$
\omega^{(\lambda)}(Q)=\omega^{(\lambda)}\left(F_{\lambda}(Q)\right)
$$

Proof. By (3.13) and (3.20) the following limit exists in the $C^{*}$ norm of

$$
\lim _{\Lambda \rightarrow \infty} F_{\lambda}^{(\Lambda)}(Q)=F_{\lambda}(Q)
$$

By (4.6), it suffices to show the existence of limit (4.9) for $Q$ in $\mathscr{B} \cap \mathscr{A}_{\text {loc }}$.

Consider a weak accumulation point of $\lim \omega_{\Lambda}^{(\lambda)}(\cdot)$. This limit is an invariant $\Lambda \rightarrow \infty$

ground state of $H(\lambda)$. By Corollary 4.2 , the limit state is unique on $\mathscr{B}$, thus we have existence of limit. (q.e.d)

We may also use (instead of (4.6)),

$$
\omega_{\Lambda}^{(\lambda)}\left(Q_{1}^{*} Q_{2}\right)=\omega_{\Lambda}^{(\lambda)}\left(F\left(Q_{1}\right)^{*} F\left(Q_{2}\right)\right) .
$$


Then

$$
\omega^{(\lambda)}\left(Q_{1}^{*} Q_{2}\right)=\omega^{(\lambda)}\left(F\left(Q_{1}\right)^{*} F\left(Q_{2}\right)\right) .
$$

Lemma 4.4. Let satisfy the assumption of Proposition 4.3. Let $\left\{\pi_{\lambda}(\cdot) \Omega_{\lambda} \mathscr{H}_{\lambda}\right\}$ be the G.S.N. triple for $\omega^{(\lambda)}$. Then

(i) $\Omega_{\lambda}$ is cyclic and separating for $\pi_{\lambda}(\mathscr{B})$,

(ii) $\pi_{\lambda}(\mathscr{B})^{\prime \prime}$ is maximally abelian in $\mathscr{B}\left(\mathscr{H}_{\lambda}\right)$.

Proof. (i) is obvious by (4.12) and commutativity of $\mathscr{B}$, (ii) follows from Lemma 4.3 .15 of [2]. (q.e.d.)

Lemma 4.5. Let $\mathscr{A}_{j}$ be the $C^{*}$ subalgebra generated by $\mathscr{B}$ and $\sigma_{x}^{(j)}$. Let $\tilde{\omega}$ be an invariant $j$ symmetric ground state of $H(\lambda)$. Suppose that $\lambda$ satisfies the assumption of Proposition 4.3. Then

$$
\tilde{\omega}(Q)=\omega^{(\lambda)}(Q) \text { for } Q \text { in } \mathscr{A}_{j} \text {. }
$$

Proof. Consider $Q=\left\{1 \pm \sigma_{z}(A)\right\} \sigma_{x}^{(j)}$, where $A$ does not contain $j$. We see that $e(1 / \lambda, \delta / \lambda)$ is analytic in a neighbourhood of $\delta=0$ and $E(H(1 / \lambda, \delta / \lambda))=e(1 / \lambda, \delta / \lambda)$ in Sect. 3. We also have

$$
\left.\frac{\partial e\left(\frac{1}{\lambda} \frac{\delta}{\lambda}\right)}{\partial \delta}\right|_{\delta=0}=\left.\lim _{\Lambda \rightarrow \infty} \frac{\partial e_{\Lambda}\left(\frac{1}{\lambda} \frac{\delta}{\lambda}\right)}{\partial \delta}\right|_{\delta=0},
$$

because of the following identity:

$$
\left.\frac{\partial e_{\Lambda}\left(\frac{1}{\lambda} \frac{\delta}{\lambda}\right)}{\partial \delta}\right|_{\delta=0}=\frac{1}{2 \pi i} \oint \frac{d z}{(\delta-z)^{2}} e_{\Lambda}\left(\frac{1}{\lambda} \frac{z}{\lambda}\right)
$$

The formula analogue to (2.4) for the periodic Hamiltonian $H_{\Lambda}(\lambda)$ is valid, so we have

$$
\left.\frac{\delta e_{\Lambda}\left(\frac{1}{\lambda} \frac{\delta}{\lambda}\right)}{\partial \delta}\right|_{\delta=0}=\omega_{\Lambda}^{(\lambda)}(Q) .
$$

$\left(\psi_{\Lambda}(\varepsilon, \delta)\right.$ is the ground state vector even if $\delta$ is negative but small by the continuity of $\psi_{\Lambda}(\varepsilon, \delta)$ in $\delta$.) Combined with (4.14), (4.16), (2.9a) leads to

$$
\tilde{\omega}\left(\left(1 \pm \sigma_{z}(A)\right) \sigma_{z}^{(j)}\right) \leqq \omega^{(\lambda)}\left(\left(1 \pm \sigma_{z}(A)\right) \sigma_{x}^{(j)}\right) .
$$

But we already know

$$
\tilde{\omega}\left(\sigma_{x}^{(j)}\right)=\omega^{(\lambda)}\left(\sigma_{x}^{(j)}\right)
$$

An arbitrary $Q$ in $\mathscr{A}_{j} \cap \mathscr{A}_{\text {loc }}$ is a linear combination of $\sigma_{x}^{(j)}, \sigma_{z}(A)$ and $\sigma_{z}(B) \sigma_{x}^{(j)}$. By $j$ symmetry of $\tilde{\omega}$, the proof of the lemma is complete. (q.e.d)

Lemma 4.6. Let $\tilde{\omega}, \lambda$ satisfy the condition of Lemma 4.5. Let $(\tilde{\pi}(\cdot) \tilde{\Omega} \mathscr{H})$ be the G.N.S. triple associated $\tilde{\omega}$. Then $\tilde{\pi}(\mathscr{B}) \tilde{\Omega}$ is dense in $\mathscr{H}$. 
Proof. It suffices to show that $\overline{\tilde{\pi}(\mathscr{B}) \widetilde{\Omega}}$ is invariant by $\tilde{\pi}\left(\sigma_{x}^{(k)}\right)$ for any $k$ in $\mathbb{Z}^{d}$. We set

$$
\mathscr{H}_{j}=\overline{\tilde{\pi}\left(\sigma_{j}\right) \widetilde{\Omega}} .
$$

In Lemma 4.5 , we see the representation of $\mathscr{A}_{j}$ on $\mathscr{H}_{j}$ is unitarily equivalent to that of $\mathscr{A}_{j}$ on $\mathscr{H}_{j}$ (the G.N.S. space associated with $\left.\omega^{(\lambda)}(\cdot)\right)$. As $\Omega_{\lambda}$ is cyclic for $\pi_{\lambda}(\mathscr{B}), \tilde{\Omega}$ is cyclic for $\tilde{\pi}(\mathscr{B})$ in $\mathscr{H}_{j}$. In particular $\overline{\tilde{\pi}(\mathscr{B}) \widetilde{\Omega}}$ is invariant by $\tilde{\pi}\left(\sigma_{x}^{(j)}\right)$. By translation invariance $\overline{\tilde{\pi}(\mathscr{B}) \widetilde{\Omega}}$ is invariant by $\tilde{\pi}\left(\sigma_{x}^{(k)}\right)$ for any $k$ in $\mathbb{Z}^{d}$. (q.e.d.)

Lemma 4.7. Let $\tilde{\omega}, \lambda$ satisfy the condition of Lemma 4.5. Then $\tilde{\omega}=\omega^{(\lambda)}$, i.e. the $j$-symmetric invariant ground state of $H(\lambda)$ is unique.

Proof. We prove

$$
\tilde{\omega}\left(\sigma_{z}(A) \sigma_{x}(B)\right)=\tilde{\omega}\left(F_{\lambda}\left(\sigma_{z}(A) \sigma_{x}(B)\right)\right) .
$$

By Corollary 4.2 and the fact that $F_{\lambda}\left(\sigma_{z}(A) \sigma_{x}(B)\right)$ is in $\mathscr{B},(4.19)$ implies the claim of lemma.

We show (4.19) by induction of $\# B$. For $\# B=1$, we have for $Q$ in $\mathscr{B} \cap \mathscr{A}_{\text {loc }}$,

$$
\tilde{\omega}\left(Q \sigma_{x}(B)\right)=\tilde{\omega}\left(F_{\lambda}\left(Q \sigma_{x}(B)\right)\right.
$$

by $j$-symmetry and Lemma 4.5. Suppose (4.20) is verified for $\# B<n-1$. Then by (4.5), Lemma 4.6 we have

$$
\tilde{\pi}\left(\sigma_{x}(B)\right) \tilde{\Omega}=\tilde{\tau}\left(F_{\lambda}\left(\sigma_{x}(B)\right)\right) \tilde{\Omega} .
$$

Then for $Q$ in $\mathscr{B}$,

$$
\begin{aligned}
\tilde{\omega}\left(Q \sigma_{x}(B \cup k)\right) & =\left(\tilde{\Omega}, \tilde{\pi}\left(Q \sigma_{x}^{(k)}\right) \tilde{\pi}\left(F_{\lambda}\left(\sigma_{x}(B)\right)\right) \tilde{\Omega}\right) \\
& =\left(\tilde{\Omega}, \tilde{\pi}(Q) \tilde{\pi}\left(\sigma_{x}^{(k)} F_{\lambda}\left(\sigma_{x}(B)\right) \sigma_{x}^{(k)-1}\right) \tilde{\pi}\left(\sigma_{x}^{(k)}\right) \tilde{\Omega}\right) .
\end{aligned}
$$

However $\sigma_{x}^{(k)} F\left(\sigma_{x}(B)\right) \sigma_{x}^{(k)-1}$ is in $\mathscr{B}$, thus

$$
\begin{aligned}
(4.12) & =\left(\tilde{\Omega}, \tilde{\pi}(Q) \tilde{\pi}\left(\sigma_{x}^{(k)} F_{\lambda}\left(\sigma_{x}(B) \sigma_{x}^{(k)-1}\right) \tilde{\pi}\left(F_{\lambda}\left(\sigma_{x}^{(k)}\right)\right) \tilde{\Omega}\right)\right. \\
& =\left(\Omega_{\lambda}, \pi_{\lambda}(Q) \pi_{\lambda}\left(\sigma_{x}^{(k)} F_{\lambda}\left(\sigma_{x}(B)\right) \sigma_{x}^{(k)-1}\right) \pi_{\lambda}\left(F_{\lambda}\left(\sigma_{x}^{(k)}\right) \Omega_{\lambda}\right) .\right.
\end{aligned}
$$

(We have used Corollary 4.2.) We trace back to (4.21) replacing $\tilde{\Omega}, \tilde{\pi}(\cdot)$ by $\Omega_{\lambda} \pi_{\lambda}(\cdot)$,

$$
(4.22)=\omega^{(\lambda)}\left(Q \sigma_{x}(B \cup\{k\})\right)=\omega^{(\lambda)}\left(F_{\lambda}\left(Q \sigma_{x}(B \cup\{k\})\right) .\right.
$$

Proof of Theorem 1.3. The centre of $\pi_{\lambda}(\mathscr{A})^{\prime \prime}$ is contained in $\pi_{\lambda}(\mathscr{B})^{\prime \prime}$ by Lemma 4.4. As $\sigma_{z}^{(j)}$ is diagonal, any selfadjoint element of $\mathscr{B}$ is $j$ invariant. Let $J$ be the antiunitary defined by

$$
J \pi_{\lambda}(Q) \Omega_{\lambda}=\pi_{\lambda}(j(Q)) \Omega_{\lambda}
$$

It is easy to see

$$
J \pi_{\lambda}(Q) J=\pi(j(Q))
$$

Then any selfadjoint element of $\pi_{\lambda}(\mathscr{B})^{\prime \prime}$ is $j$ invariant in the sense that

$$
Q=J Q J \quad \text { if } \quad Q=Q^{*} \in \pi_{\lambda}(\mathscr{B})^{\prime \prime} .
$$


Let $\tilde{\omega}$ be an invariant ground state. Then by Lemma 4.7. We have

$$
\omega^{(\lambda)}(Q)=\frac{\tilde{\omega}(Q)+\overline{\tilde{\omega}(j(Q))}}{2} .
$$

Thus there exists a projection $p$ in the centre of $\pi_{\lambda}(\mathscr{A})^{\prime \prime}$ such that

$$
\omega(Q)=\frac{\left(\Omega_{\lambda}, P \pi_{\lambda}(Q) \Omega_{\lambda}\right)}{\left(\Omega_{\lambda}, P \Omega_{\lambda}\right)} .
$$

Then, $\tilde{\omega}(\cdot)$ is $j$ symmetric by (4.25), because

$$
\begin{aligned}
\tilde{\omega}(Q) & =\frac{\left(J \Omega_{\lambda}, P \pi_{\lambda}(Q) J \Omega_{\lambda}\right)}{\left(\Omega_{\lambda}, P \Omega_{\lambda}\right)}=\frac{\left(J P \pi_{\lambda}(Q) J \Omega_{\lambda}, \Omega_{\lambda}\right)}{\left(\Omega_{\lambda}, P \Omega_{\lambda}\right)} \\
& =\frac{\left(P \pi_{\lambda}(j(Q)) \Omega_{\lambda}, \Omega_{\lambda}\right)}{\left(\Omega_{\lambda}, P \Omega_{\lambda}\right)}=\tilde{\omega}\left(j\left(Q^{*}\right)\right)=\overline{\tilde{\omega}(j(Q)) .}
\end{aligned}
$$

By Lemma $4.7 \omega_{\lambda}=\tilde{\omega}$.

Remark 4.8. We proved $\omega_{\lambda}$ is pure because the Gibbs state we considered is ergodic and our analysis shows the centre of $\pi_{\lambda}(\mathscr{A})^{\prime \prime}$ coincides with $\pi_{\lambda}(\mathscr{A})^{\prime}$ by Lemma 4.4.

\section{References}

1. Bratteli, O., Kishimoto, A., Robinson, D.: Ground states of quantum spin systems. Commun. Math. Phys. 64, 41-48 (1978)

2. Bratteli, O., Robinson, D.: Operator algebras and quantum statistical mechanics. Berlin, Heidelberg, New York: Springer 1981

3. Kato, T.: Perturbation theory for Linear operators. Berlin, Heidelberg, New York: Springer,

4. Kirkwood, J. R., Thomas, L.: Expansions and phase transitions for ground states of quantum Ising lattice systems. Commun. Math. Phys. 88, 569-580 (1983)

Communicated by M. Aizenman

Received November 29, 1988; in revised form May 16, 1989 
